\title{
A low-voltage three-axis electromagnetically actuated micromirror for fine alignment among optical devices
}

\author{
Il-Joo Cho and Euisik Yoon \\ Department of Electrical Engineering and Computer Science, University of Michigan, 1301 Beal Ave, \\ Ann Arbor MI 48109, USA \\ E-mail: ijcho@umich.edu
}

Received 11 February 2009, in final form 21 May 2009

Published 9 July 2009

Online at stacks.iop.org/JMM/19/085007

\begin{abstract}
In this paper, a new three-axis electromagnetically actuated micromirror structure has been proposed and fabricated. It is electromagnetically actuated at low voltage using an external magnetic field. The main purpose of this work was to obtain a three-axis actuated micromirror in a mechanically robust structure with large static angular and vertical displacement at low actuation voltage for fine alignment among optical components in an active alignment module as well as conventional optical systems. The mirror plate and torsion bars are made of bulk silicon using a SOI wafer, and the actuation coils are made of electroplated Au. The maximum static deflection angles were measured as $\pm 4.2^{\circ}$ for $x$-axis actuation and $\pm 9.2^{\circ}$ for $y$-axis actuation, respectively. The maximum static vertical displacement was measured as $\pm 42 \mu \mathrm{m}$ for $z$-axis actuation. The actuation voltages were below $3 \mathrm{~V}$ for all actuation. The simulated resonant frequencies are several $\mathrm{kHz}$, and these imply that the fabricated micromirror can be operated in sub-millisecond order. The measured radius of curvature (ROC) of the fabricated micromirror is $7.72 \mathrm{~cm}$, and the surface roughness of the reflector is below $1.29 \mathrm{~nm}$ which ensure high optical performance such as high directionality and reflectivity. The fabricated micromirror has demonstrated large actuated displacement at low actuation voltage, and it enables us to compensate a larger misalignment value when it is used in an active alignment module. The robust torsion bar and lifting bar structure formed by bulk silicon allowed the proposed micromirror to have greater operating stability. The additional degree of freedom with $z$-axis actuation can decrease the difficulty in the assembly of optical components and increase the coupling efficiency between optical components.
\end{abstract}

(Some figures in this article are in colour only in the electronic version)

\section{Introduction}

During the past few years, small optical systems have emerged not only for replacing current large optical systems but also for introducing new optical systems. In these small optical systems, alignment among optical components is critical to achieve a low signal loss since a small misalignment among them can induce a large signal loss in these systems [1]. To minimize the misalignment, several passive alignment methods were proposed such as optical bench [2]. However, fabrication errors may cause unacceptable coupling efficiency in the case of passive alignment schemes. Therefore, active alignment has been proposed using a MEMS micromirror [3].

To achieve a large compensation value and high coupling efficiency between optical components in an active alignment, the micromirror should have a large static actuation angle. Previously, several MEMS micromirrors have been introduced for various applications such as optical switch [4], optical scanner [5], projection display [6] and alignment module, etc. However, most of the micromirrors reported to date have used electrostatic force actuation [7-10]. In the case of electrostatic actuation, high actuation voltage is required for a large deflection angle, and most of them use the resonant 


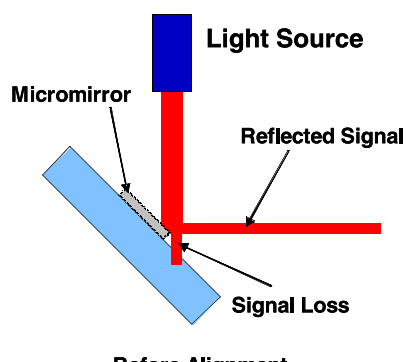

Before Alignment

(a)

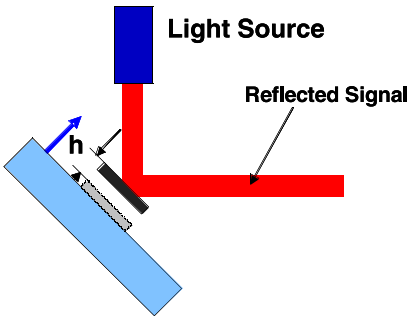

After Alignment

(b)

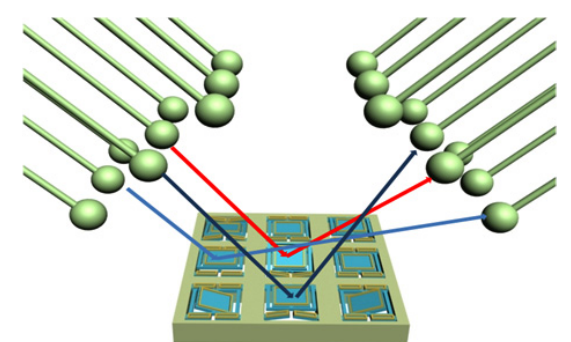

(c)

Figure 1. (a) Schematic diagram showing signal loss from the misalignment between the light source and the micromirror. (b) Schematic diagram of active alignment between the light source and the target position using the micromirror with $z$-axis actuation. (c) An example application of the proposed micromirror array.

mode to obtain this large deflection angle. This makes it difficult to be used as a micromirror in an active alignment system. In contrast, electromagnetic actuation can generate a relatively large force with a lower voltage even in a static mode, and several micromirrors reported previously used this actuation force [11-14]. This large actuation force enables a large static deflection angle at low actuation voltage, and this large static actuation angle is much more useful for applying it to an active alignment module due to the greater misalignment it can compensate. Also, this large electromagnetic force actuation enables the micromirrors to have mechanically robust structures which ensure reliable operation in static mode operation. However, the previously reported electromagnetically actuated micromirrors only rotate around two axes (the $x$-axis and $y$-axis). Therefore, the light path between the light source and the micromirror should be quite accurately pre-aligned in most optical systems, even in active alignment modules adapting this two-axis actuated micromirror. It cannot have a full freedom of active alignment after they are assembled because micromirrors can only be actuated around the two axes and cannot align the light path between the light source and the micromirror as shown in figure $1(a)$. Therefore, it is required that the micromirror has $z$-axis actuation in order to align the light path between the light source and the mirror plate as shown in figure $1(b)$. Previously, several micromirrors which have $z$-axis actuation as well as $x$-axis and $y$-axis actuation have been introduced. However, electrostatically actuated micromirrors require high actuation voltage over $100 \mathrm{~V} \quad[15,16]$ and thermally actuated micromirror shows slow response time around $10 \mathrm{~ms}$ [17].

In this work, we have proposed a low-voltage threeaxis electromagnetically actuated micromirror. The proposed

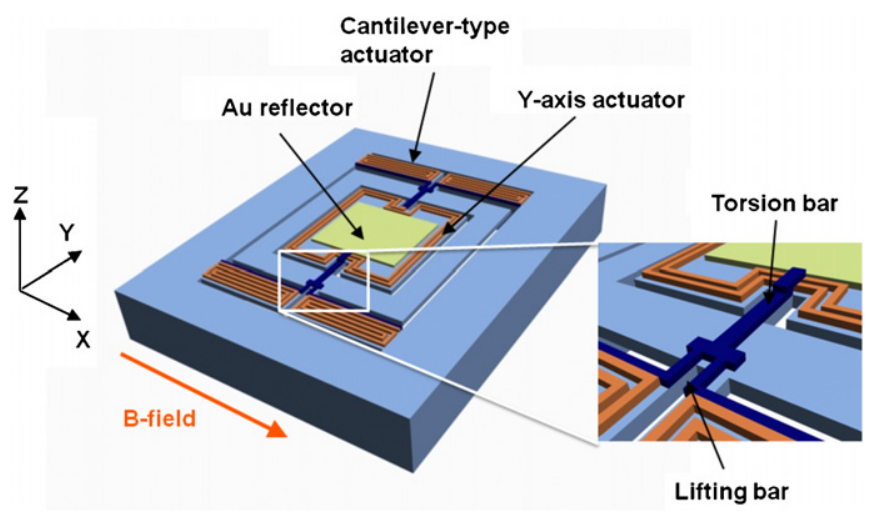

Figure 2. Schematic diagram of the proposed micromirror.

micromirror can actuate along the $z$-axis as well as around the $x$-axis and the $y$-axis. This additional degree of freedom with $z$-axis actuation can decrease the difficulty in the assembly of optical components and increase the coupling efficiency between optical components by aligning the light path between the light source and the micromirror in an active alignment module. Furthermore, this proposed three-axis actuated micromirror can be applied to complicated optical systems such as optical switch matrix as shown in figure 1(c) as well as simple optical systems such as the active alignment module and scanning display, etc. The proposed micromirror also uses electromagnetic actuation and we have used an external magnetic field to obtain large force at low voltage and at low power consumption.

In this paper, design, fabrication and experimental results of the three-axis electromagnetically actuated micromirrors will be presented.

\section{Design and simulation}

The proposed micromirror is actuated by Lorentz force using an external electromagnetic field as shown in figure 2. The micromirror structure consists of a gold-coated mirror plate, incorporated actuation coils, frame and cantilever-type actuators with actuation coils. The cantilever-type actuators are connected to the frame using lifting bars, and the frame is connected to the mirror plate using torsion bars. The mechanical structure is made of silicon. The coils are electroplated gold integrated on the mirror plate and cantilevertype actuators. There are two types of actuators in the micromirror. The actuator coils integrated on the mirror plate is used for $y$-axis actuation and the other coil actuators integrated on the cantilevers are used for $x$-axis and $z$-axis actuation. The coils on the mirror plate are connected to the pads on the frame through the torsion bars and lifting bars. Theses coils effectively increase the total thickness of torsion bars and lifting bars in the proposed structure and increasing the robustness.

Figure 3 show the simulation results using ANSYS and they illustrate the operation principle of the proposed micromirror. The operation of the micromirror is determined by the direction of the current flowing in the coils on the mirror 


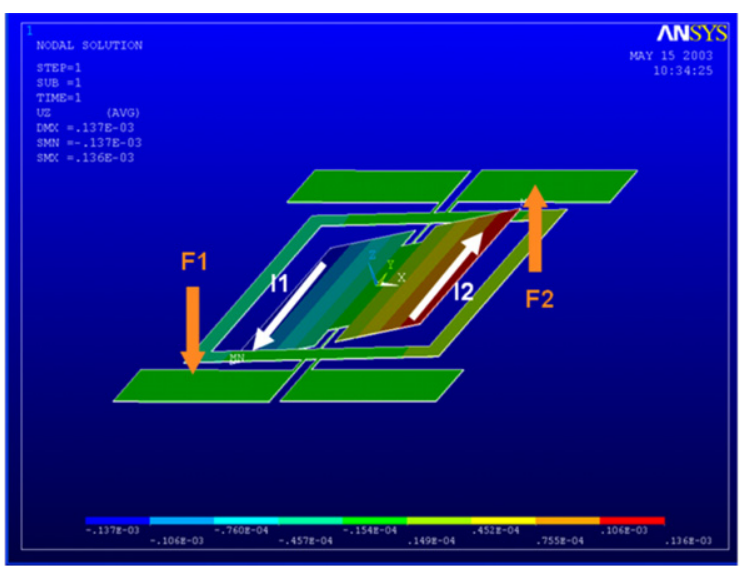

(a)

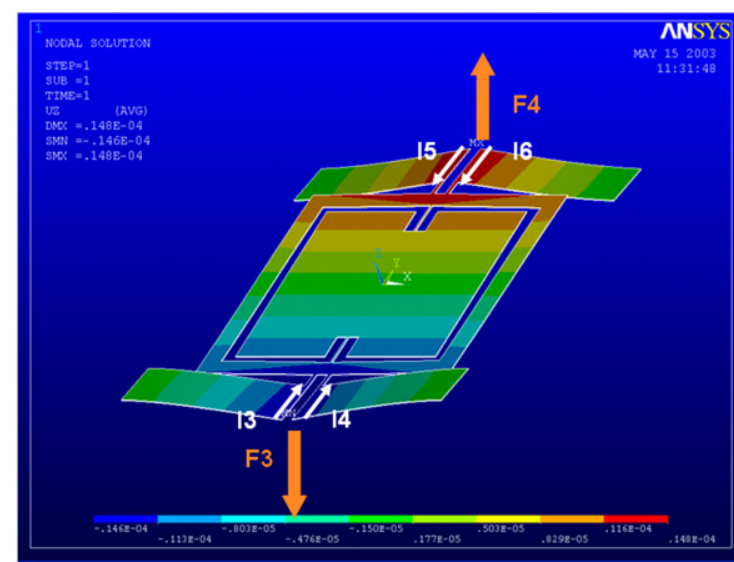

(b)

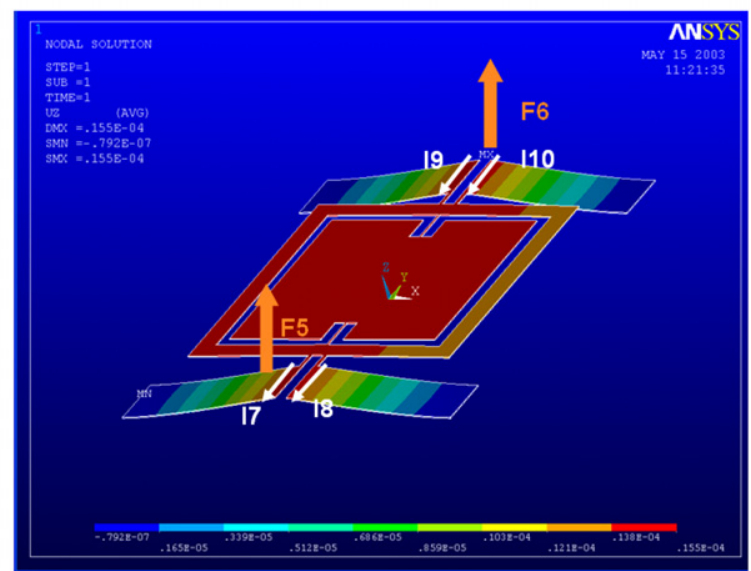

(c)

Figure 3. FEM simulation results using ANSYS showing the displacement of the mirror plate with generated forces: $(a) y$-axis actuation mode with a resonant frequency of $3.391 \mathrm{kHz},(b) x$-axis actuation mode with a resonant frequency of $5.369 \mathrm{kHz}$ and $(c) z$-axis actuation mode with a resonant frequency of $7.258 \mathrm{kHz}$.

plate and the cantilever actuators. Among these coils, only the current component in the coils perpendicular to the magnetic field can induce a Lorentz force. In the case of $y$-axis actuation, the coils on the mirror plate induce the Lorentz force as shown in figure 3(a).

The coils which flow a current, $I_{1}$, induce an upward force, $F_{1}$, while the coils which flow a current, $I_{2}$, induce a downward force, $F_{2}$. These forces enable the mirror plate to rotate around the $y$-axis by the inner torsion bars as a central axis. In the case of $x$-axis and $z$-axis actuation, the coils on the cantilever actuators induce the Lorentz force. Figure 3(b) illustrates the $x$-axis actuation principle. In this case, the coils which flow current, $I_{3}$ and $I_{4}$, induce upward force, $F_{3}$, and this force lifts up the pair of cantilevers. The lifted cantilevers accordingly lift the frame. The other pair of cantilevers moves downward by the Lorentz force, $F_{4}$, induced from current $I_{5}$ and $I_{6}$, making the frame move downward. From this cantilever actuation, the frame rotates around the $x$-axis since two pairs of cantilevers actuate the same distance in opposite directions, respectively. If these two pairs of cantilevers are actuated in the same direction, the micromirror can move along the $z$-axis as shown in figure $3(c)$ because $F_{5}$ and $F_{6}$ are in the same direction. If opposite direction of current is applied on coils, the micromirror can be actuated in the opposite direction.
To predict the relationship between actuation current and displacement of the micromirror, the actuation is simulated using ANSYS with a simplified model. Figure 3 shows the actuation modes of each direction simulated using ANSYS. The resonant frequency of $x$-axis actuation is $5.369 \mathrm{kHz}$, that of $y$-axis actuation is $3.391 \mathrm{kHz}$ and that of $z$-axis actuation is $7.258 \mathrm{kHz}$, respectively. These resonant frequencies allow this micromirror to be operated in sub-milliseconds.

The detailed dimensions of the fabricated structure are summarized in table 1 .

\section{Fabrication}

The fabrication processes of the proposed micromirror are shown in figure 4 . Starting material is a SOI wafer with the top silicon of $5 \mu \mathrm{m}$ and the buried oxide of $1 \mu \mathrm{m}$. The thickness of the top silicon directly determines the thickness of the proposed micromirror. Thermal oxide of $5000 \AA$ is grown and used for a dry etch mask in etching top silicon. Also, LPCVD silicon nitride is deposited for an etch mask of silicon anisotropic etch. Mirror frames and mirror plates are defined at the top silicon by reactive ion etching (RIE) using the thermal oxide as an etch mask (figure 4(b)). Then, $\mathrm{Cr} / \mathrm{Au}$ is deposited for a seed metal layer for electroplating $\mathrm{Au}$ as 
Table 1. Summarized dimensions of the proposed micromirror.

\begin{tabular}{|c|c|c|c|}
\hline Mirror plate & \multicolumn{3}{|c|}{$900 \mu \mathrm{m} \times 900 \mu \mathrm{m}$} \\
\hline Torsion-bar length & \multicolumn{3}{|c|}{$200 \mu \mathrm{m}$} \\
\hline Torsion-bar width & \multicolumn{3}{|c|}{$15 \mu \mathrm{m}$} \\
\hline Torsion-bar thickness & \multicolumn{3}{|c|}{$10 \mu \mathrm{m}($ silicon $5 \mu \mathrm{m}+\mathrm{Au}$ coil $5 \mu \mathrm{m})$} \\
\hline Lifting-bar length & \multicolumn{3}{|c|}{$100 \mu \mathrm{m}$} \\
\hline Lifting-bar width & \multicolumn{3}{|c|}{$20 \mu \mathrm{m}$} \\
\hline Lifting-bar thickness & \multicolumn{3}{|c|}{$10 \mu \mathrm{m}$} \\
\hline Coil width & \multicolumn{3}{|c|}{$15 \mu \mathrm{m}$} \\
\hline Coil to coil spacing & \multicolumn{3}{|c|}{$10 \mu \mathrm{m}$} \\
\hline Coil thickness & \multicolumn{3}{|c|}{$5 \mu \mathrm{m}$} \\
\hline $\begin{array}{l}\text { Resonant frequency } \\
\text { (simulated) }\end{array}$ & $\begin{array}{l}5.369 \mathrm{kHz} \\
(x \text {-axis actuation })\end{array}$ & $\begin{array}{l}3.391 \mathrm{kHz} \\
\text { (y-axis actuation) }\end{array}$ & $\begin{array}{l}7.258 \mathrm{kHz} \\
\text { (z-axis actuation) }\end{array}$ \\
\hline
\end{tabular}

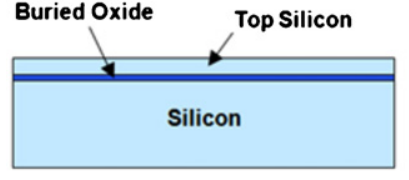

(a)

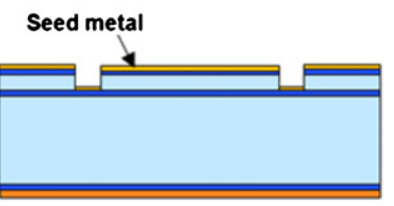

(c)



(e)

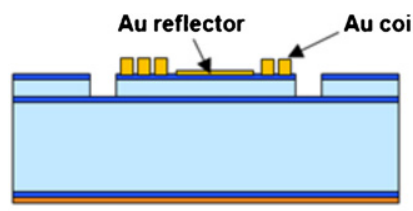

$(g)$

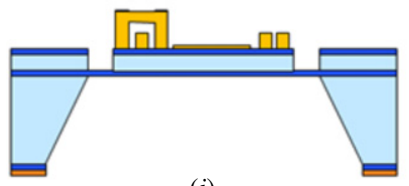

(i)

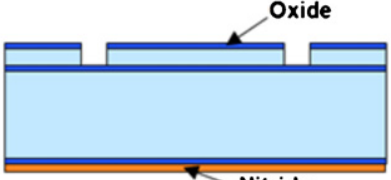

(b) Nitride

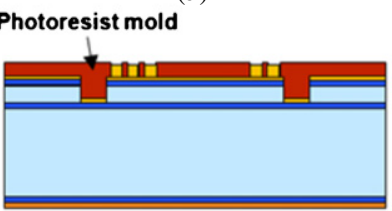

(d)

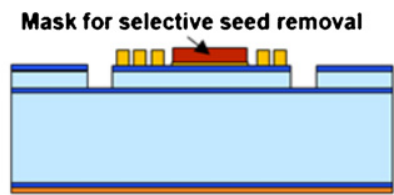

$(f)$

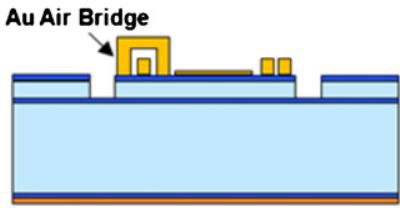

(h)
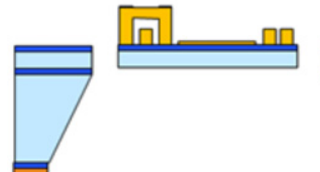

$(j)$
Figure 4. Process flow of the proposed structure: $(a)$ starting SOI wafer, $(b)$ mirror structure defined by top silicon dry etch, $(c)$ seed metal deposition for electroplating, $(d)$ lower coil electroplating with a photoresist mold, $(e)$ photoresist mold removal,

$(f)$ photoresist mask patterning for the Au reflector using selective seed removal, $(g)$ lower coil with the Au reflector, $(h)$ air bridge coil electroplated with photoresist as a gap material, $(i) \mathrm{Si}$ anisotropic etch in $\mathrm{KOH}$ and $(j)$ buried oxide removal.

shown in figure 4(c). Next, lower Au coils are electroplated as shown in figure $4(d)$. The $5 \mu \mathrm{m}$ thick Au coils are electroplated using $10 \mu \mathrm{m}$ thick AZ9260 photoresist as a mold. Because these electroplated Au coils present low intrinsic stress below $20 \mathrm{MPa}$, we can obtain a flat micromirror structure. In this process, the Au seed layer is also used as a reflector material on the mirror plate to reduce process complexity. Therefore, the seed layer is selectively removed with the photoresist mask as shown in figure $4(f)$. After electroplating the lower Au coils, photoresist is coated for a sacrificial layer to make an air gap and patterned for a contact. Following this, another Au layer is deposited as a seed layer. With photoresist as a mold, the bridge metal is electroplated as shown in figure $4(h)$. These bridge metals have a role of connecting metal lines. Then, the photoresist used for the mold is removed in acetone.

Next, silicon nitride is patterned on the backside with photoresist for an etch mask during anisotropic silicon etch. Using the buried oxide as an etch stop, silicon is etched from the backside in $\mathrm{KOH}$ using silicon nitride as an etch mask (figure 4(i)). After removing the buried oxide, the complete micromirrors are released as shown in figure $4(j)$.

Figure 5 shows the pictures of the fabricated micromirror. Figure 5(a) shows the overall view of the fabricated micromirror. The pictures show that the fabricated micromirror has a stress-free structure which is obtained from a thick silicon structure and an electroplated $\mathrm{Au}$ coil with low stress. The enlarged view of the mirror plate is shown in figure $5(b)$. On the center of the mirror plate a thin gold film is deposited in order to increase the reflectivity of light at the mirror plate. Figure 5(c) shows the cantilever-type actuator used for $x$-axis actuation and $z$-axis actuation. The air bridge metal is used for electrical connection between two points while preventing wire crossing. Figure $5(d)$ shows the picture of a torsion bar. Metal lead wires are electroplated on the torsion bars for electrical connections. The total thickness of the torsion bars and lifting bars will be the sum of the top silicon thickness and the lead wire thickness, which is about $10 \mu \mathrm{m}$. These thick torsion bars enable mechanically stable operation under a large electromagnetic force induced from an external magnetic field.

\section{Measurement and results}

To measure deflection angles, current is applied to the $\mathrm{Au}$ coils. The diced micromirror is mounted on a PCB board using epoxy, and the metal pads on the micromirror and $\mathrm{PCB}$ board are connected by bonding wires. The actuation current is applied to the micromirror via the PCB board. Afterward, it is inserted between permanent magnets in a way to apply a magnetic field parallel to the $x$-axis of the micromirror as proposed in figure 2 . The magnetic field intensity is measured at the actuation point using a Gauss meter (F W Bell 5080). 


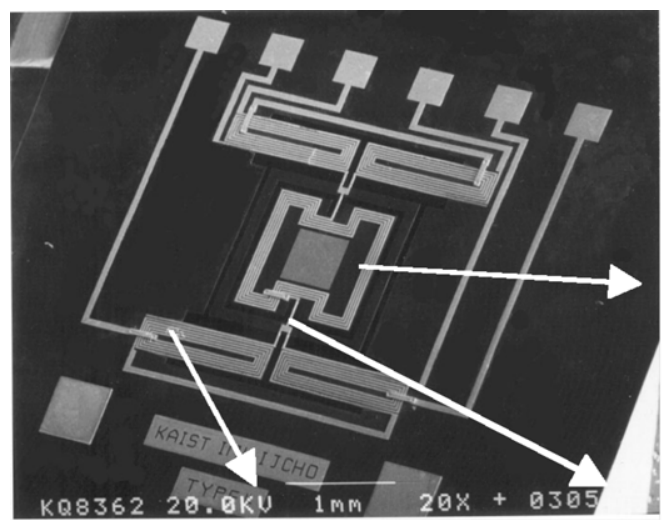

(a)



(c)

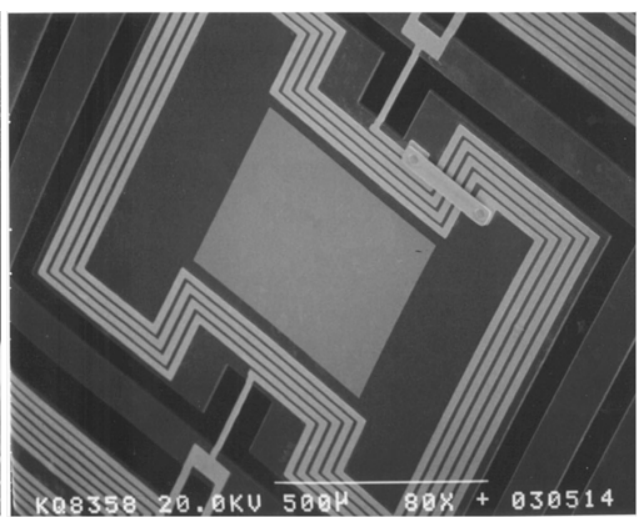

(b)

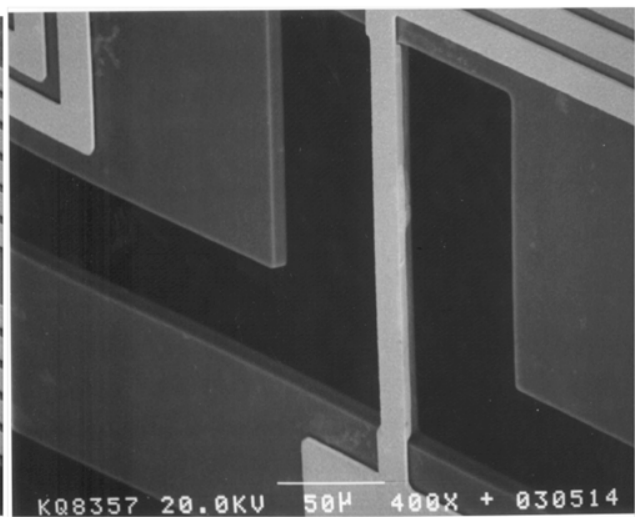

(d)

Figure 5. SEM pictures of the fabricated mirror structure.

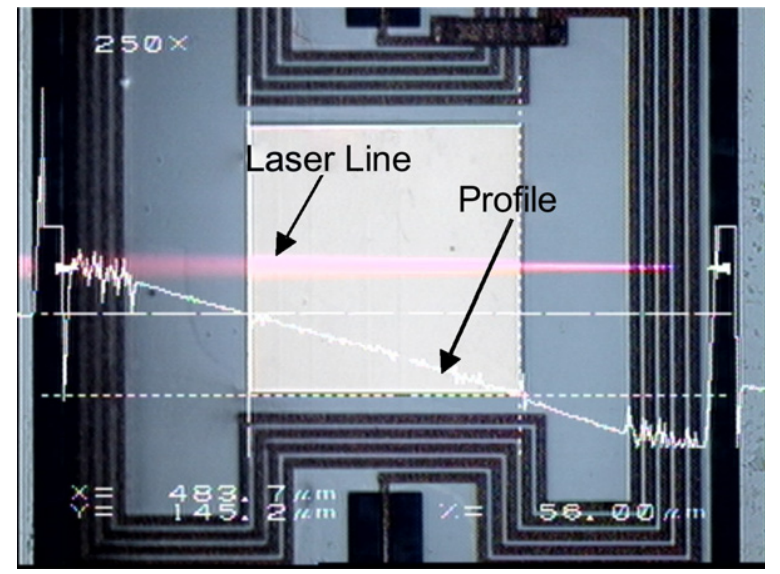

Figure 6. Picture of the rotated micromirror using a 3D profiler.

The measured magnetic field intensity is $0.16 \mathrm{~T}$, which is equal to 1600 Gauss.

\subsection{The rotational angle and z-axis movement}

The rotational angle and the vertical displacement of the actuated micromirror are measured using a 3D laser profiler (Keyence VF-7500). This laser profiler scans the sample surface with a focused laser line and displays a 3D profile as shown in figure 6 . To measure the actuated displacement, the actuated mirror plate is scanned with a focused laser line from the $3 \mathrm{D}$ profiler, and the actuation angle is obtained by measuring the difference in the height between the left side and the right side of the mirror plate. As a result of rotation, the left part of the mirror plate is seen as dark while the other side is seen as bright, and the focus of the laser line is blur on the left side while focused on the right side as shown in figure 6 . The white line in figure 6 is the profile of the actuated micromirror. The profile is seen to be inclined on the mirror plate, and the slope of the profile increases with the rotation angle of the micromirror. The rotation angle can also be confirmed from the dimensional change in the sidewall of the mirror plate. The $z$-axis vertical movement of the micromirror is obtained by measuring the $3 \mathrm{D}$ profile of the $z$-axis actuated micromirror comparing to the fixed substrate.

Actuation is measured by applying a constant current. The applied voltage can also be calculated with applied current and the $\mathrm{Au}$ coil resistance. Figure 7 shows the measured deflections of the micromirror as a function of applied current for $x$-axis, $y$-axis and $z$-axis actuation, respectively.

In the case of $x$-axis actuation, the maximum static deflection angle has been measured as $4.2^{\circ}$ at an applied dc current of $115 \mathrm{~mA}$ and an actuation voltage of $3.0 \mathrm{~V}$. The total resistance of the coils on the cantilever-type actuators is $26.1 \Omega$. In the case of $y$-axis actuation, the maximum static deflection angle has been measured as $9.2^{\circ}$ at an applied dc current of $120 \mathrm{~mA}$ and an actuation voltage of $3.0 \mathrm{~V}$. 


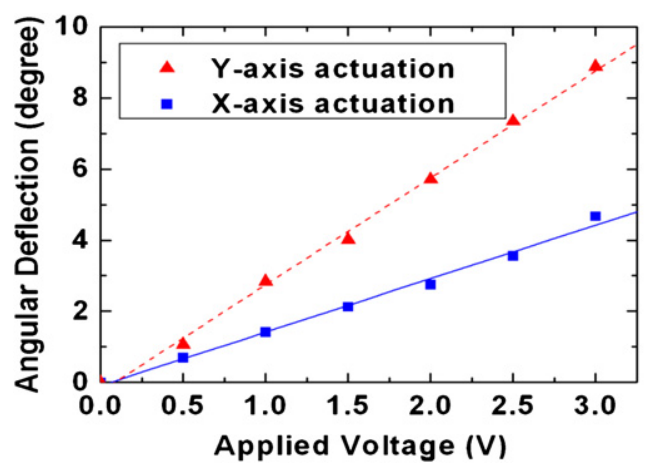

(a)

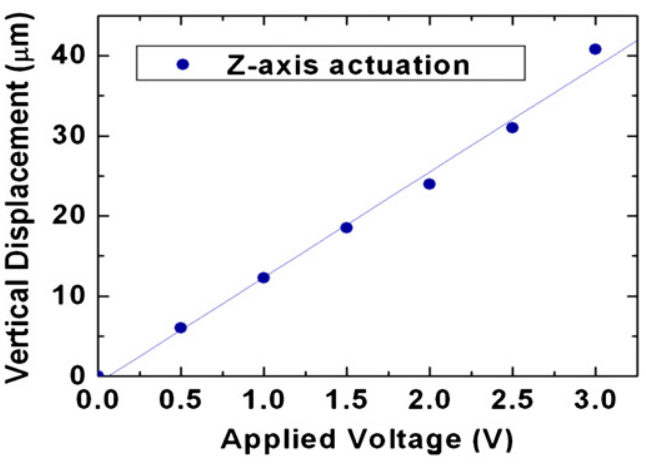

(b)

Figure 7. Measured deflection angle as a function of applied voltage: (a) $x$-axis and $y$-axis angular deflection, $(b) z$-axis displacement.
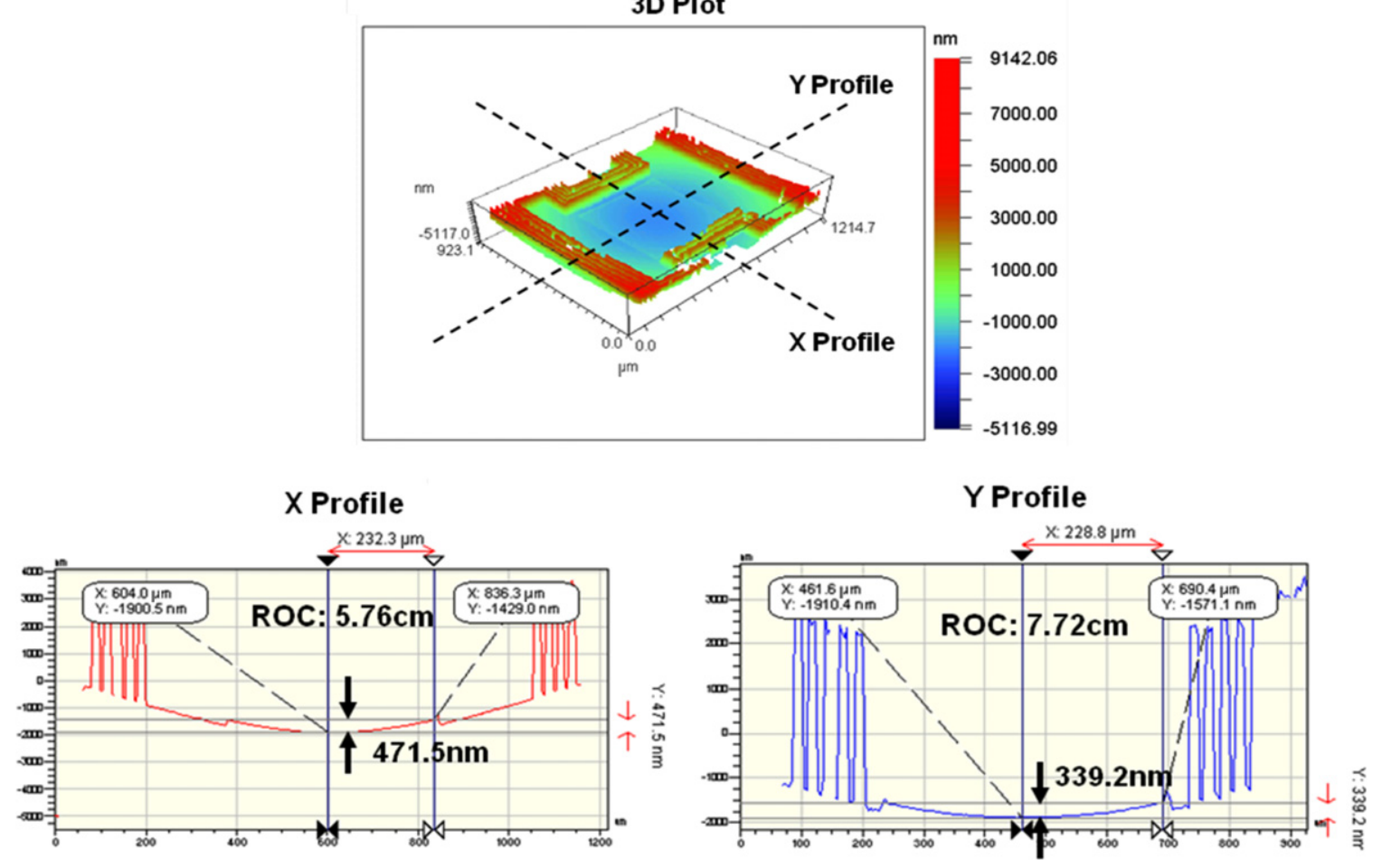

Figure 8. Measured radius of curvature (ROC) of the fabricated micromirror along the $x$-axis and the $y$-axis using a 3D surface profiler.

The total resistance of the coils for $y$-axis actuation on the mirror plate is $25.0 \Omega$. The $z$-axis vertical movement of the micromirror is $42 \mu \mathrm{m}$ at an applied dc current of $115 \mathrm{~mA}$ and an actuation voltage of $3.0 \mathrm{~V}$. The applied current is relatively large and there is a chance that this may induce electrothermal actuation. Therefore, we observed the electrothermal effect by applying current without a magnetic field. And, there was no electrothermal effect within the error range $(<0.1 \mu \mathrm{m})$. However, the actuation current should be reduced for reducing power consumption, and it can be reduced by applying higher magnetic field intensity at the actuation point. If the fabricated micromirror is packaged with a permanent magnet, the magnetic field intensity can be increased by reducing the gap between the micromirror and permanent magnets. Furthermore, the actuation current can be reduced by maximizing the turns of coils on the actuators.

\subsection{Radius of curvature (ROC)}

The radius of curvature (ROC) of a micromirror is the factor which determines the flatness of the micromirror. A large ROC value of the micromirror implies that the micromirror has a flat surface and a low signal loss. Therefore, it is an important factor for the micromirror to maintain the desired length of the light path and to reduce the signal loss. To measure the ROC of the fabricated micromirror, its surface was characterized by using a Wyko NT2000 3D optical profiler as shown in figure 8 . The measured ROC of the fabricated micromirror is $7.72 \mathrm{~cm}$ around the $y$-axis and $5.76 \mathrm{~cm}$ around the $x$-axis. These are sufficient values even for large port optical crossconnect (OXC) systems, which require a high-performance micromirror [1, 18] as well as simple optical systems. It shows that the actuator coils and the reflector integrated on the 




Figure 9. Surface roughness image of the reflector on the mirror plate using an AFM.

mirror plate have little effect on the curvature of the fabricated micromirror due to a thick silicon structure.

\subsection{Reflectivity}

The fabricated micromirror is coated with Au to increase the reflectivity of incident light. In addition to the coated material on the mirror plate, the surface roughness of the micromirror is strongly related to its reflectivity. The micromirror with high reflectivity can reduce the signal loss on the mirror plate. To measure the surface roughness of the fabricated micromirror, the surface of the mirror plate is scanned with an atomic force microscope (AFM) in the contact mode. The measured root-mean-square roughness of the mirror surface is $1.29 \mathrm{~nm}$, and this value represents that the reflectivity of the fabricated micromirror can be higher than $95 \%$ [19].

\section{Conclusions}

We have proposed and fabricated a new three-axis actuated micromirror. This fabricated micromirror operates electromagnetically using an external magnetic field. The maximum static actuation values of the three-axis actuated micromirror have been measured as $\pm 4.2^{\circ}$ at $115 \mathrm{~mA}$ for $x$-axis actuation, $\pm 9.2^{\circ}$ at $120 \mathrm{~mA}$ for $y$-axis actuation and $\pm 42 \mu \mathrm{m}$ for $z$-axis actuation, respectively. The actuation voltages are below $3.0 \mathrm{~V}$ for all actuation. The simulated resonant frequencies are several $\mathrm{kHz}$, and this implies that the fabricated micromirror can be operated in millisecond order which is sufficient for alignment. The measured ROC of the fabricated micromirror is over $5.76 \mathrm{~cm}$, and the surface roughness of the micromirror is $1.29 \mathrm{~nm}$. These values show that the signal loss of the micromirror is low enough to be applicable to large port optical cross-connect switch which requires a high-performance micromirror for its long optical path. The fabricated micromirror demonstrated large actuated static displacement at low actuation voltage. The mechanically robust torsion bar and lifting bar structure formed by bulk silicon allows the proposed micromirror to stably operate end extends the lifetime of the fabricated micromirror. The third $z$-axis actuation of the fabricated micromirror reduces the difficulty of assembly and increases the coupling efficiency of the micromirror-based active alignment module. Also, it can be used in optical systems such as optical switch, projection display and optical scanning, and it can increase system performance due to its large actuation angle and high degree of freedom with low actuation voltage. The applied current of the fabricated micromirror can be reduced by applying higher magnetic field intensity at the actuation point or by maximizing the number of turns of coils on the actuators.

\section{References}

[1] Lin L, Goldstein E and Tkach R 2000 On the expandability of free-space micromachined optical cross connects J. Lightwave Technol. 18482

[2] Kwon H, Kim S, Yee Y, Ha J, Song K, Um K, Nam H, Joo Y and $\mathrm{Bu} \mathrm{J} 2007$ Micro-optical fiber coupler on silicon bench based on microelectromechanical systems technology Japan. J. Appl. Phys. 465473

[3] Ishikawa K, Zhang J, Tuantranont A, Bright V and Lee Y 2003 An integrated micro-optical system for VCSEL-to-fiber active alignment Sensors Actuators A 103 109-15

[4] Goldstein E, Lin L, Inc T and Oceanport N 2000 Optical-layer networking with lightwave micromachines: what doservice-providers need and when do they need it? IEEE/LEOS Int. Conf. on Optical MEMS pp 27-8

[5] Kiang M, Solgaard O, Muller R and Lau K 1996 Surface-micromachined electrostatic-comb driven scanningmicromirrors for barcode scanners Micro Electro Mechanical Systems pp 192-7

[6] Wine D, Helsel M, Jenkins L, Urey H and Osborn T 2000 Performance of a biaxial MEMS-based scanner for microdisplay applications Proc. SPIE 186-96

[7] Conant R, Hagelin P, Krishnamoorthy U, Hart M, Solgaard O, Lau K and Muller R 2000 A raster-scanning full-motion video display using polysilicon micromachined mirrors Sensors Actuators A 83 291-6

[8] Bhalotra S, Mansell J, Kung H and Miller D 2000 Parallel-plate MEMS mirror design for large on-resonancedisplacement IEEE/LEOS Int. Conf. on Optical MEMS pp 93-4

[9] Toshiyoshi H and Fujita H 1996 Electrostatic micro torsion mirrors for an optical switch matrix J. Microelectromech. Syst. 5 231-7

[10] Jung I, Krishnamoorthy U and Solgaard O 2006 High fill-factor two-axis gimbaled tip-tilt-piston micromirror array actuated by self-aligned vertical electrostatic combdrives J. Microelectromech. Syst. 15 563-71

[11] Ji C and Kim Y 2003 Electromagnetic micromirror array with single-crystal silicon mirror plate and aluminum spring J. Lightwave Technol. 21584

[12] Taylor W, Bernstein J, Brazzle J and Corcoran C 2003 Magnet arrays for use in a 3-D MEMS mirror array for optical switching IEEE Trans. Magn. 39 3286-8

[13] Yalcinkaya A, Urey H, Brown D, Montague T and Sprague R 2006 Two-axis electromagnetic microscanner for high resolution displays J. Microelectromech. Syst. 15786

[14] Cho I, Yun K, Lee H, Yoon J and Yoon E 2002 A low voltage two-axis electromagnetically actuated micromirror with bulk silicon mirror plates and torsion bars IEEE Int. MEMS Conf. Tech. Dig. pp 20-4

[15] Milanovic V, Matus G and McCormick D 2004 Gimbal-less monolithic silicon actuators for tip-tilt-piston micromirror applications IEEE J. Sel. Top. Quantum Electron. 10 462-71 
[16] Tsai J, Lu L, Hsu W, Sun C and Wu M 2008 Linearization of a two-axis MEMS scanner driven by vertical comb-drive actuators J. Micromech. Microeng. 1815015

[17] Singh J, Gan T, Agarwal A and Liw S 2005 3D free space thermally actuated micromirror device Sensors Actuators A $123468-75$
[18] Chu P, Lee S, Park S, Inc T and Oceanport N 2002 MEMS: the path to large optical crossconnects IEEE Commun. Mag. 40 80-7

[19] Zou J, Balberg M, Byrne C, Liu C and Brady D 1999 Optical properties of surface micromachined mirrors with etch holes J. Microelectromech. Syst. 8 506-13 